\title{
Durée de vie et développement durable
}

Sustainability and the Lifetimes of Buildings

\section{Marc Méquignon}

\section{(2) OpenEdition}

Journals

Édition électronique

URL : http://journals.openedition.org/crau/587

DOI : $10.4000 /$ crau. 587

ISSN : 2547-5746

\section{Éditeur}

Éditions du patrimoine

\section{Édition imprimée}

Date de publication : 1 novembre 2012

Pagination : 225-232

ISBN : 978-2-7577-0108-9

ISSN : 1296-4077

Référence électronique

Marc Méquignon, «Durée de vie et développement durable », Les Cahiers de la recherche architecturale et urbaine [En ligne], 26/27 | 2012, mis en ligne le 01 novembre 2017, consulté le 19 avril 2019. URL : http://journals.openedition.org/crau/587 ; DOI : 10.4000/crau.587 
La durée de vie d'un bâtiment est un facteur clé dans une approche de développement durable. L'article présente d'abord les mécanismes qui régissent le vieillissement et la complexité de la durée de vie du bâtiment. Une deuxième partie propose synthétiquement les données disponibles permettant l'évaluation des impacts environnementaux et sanitaires. La dernière partie analyse les données et les normes, pose des questions et propose des pistes de recherche dont les objectifs permettraient des décisions plus performantes.

\section{Durée de vie et développement durable}

MARC MÉQUIGNON

En 2007, L'Ordre des architectes a rappelé que « les constructions doivent être pensées dans le long terme». De son coté, l'Assemblée générale du Conseil des architectes d'Europe a écrit "qu'une stratégie de développement durable cherchera à prolonger la vie des structures existantes ». En 2008 et 2009, l'Agence nationale de la recherche mentionne la question de la durée de vie et de son prolongement dans ses appels à projets. Enfin, l'ADEME, Agence de l'environnement et de la maîtrise de l'énergie, dans son ouvrage Qualité environnementale des bâtiments pose la question de savoir s'il faut concevoir des bâtiments jetables que l'on démolira à la première occasion de changement d'usage ou s'il faut préférer des bâtiments durables mais capables de s'adapter au cours de leur vie aux changements d'usage et de technique.

Niklaus Kohler ${ }^{1}$ déclare que l'amphithéâtre de Nîmes est une référence parfaite de construction durable qui continue d'être utilisée pour différentes applications depuis deux mille ans. À l'opposé, Nicolas Babey ${ }^{2}$ considère qu'il est souvent injustifié de

1. Niklaus Kohler, "The relevance of Green Building Challenge: an observer's perspective ", Building Research and Information, vol. 27, $n^{\circ} 4 / 5,1999$, p. 309-20.

2. Nicolas Babey, «Faut-il construire pour 30 ans », Projections, association Ecoparc, 2003. 
construire pour trente ans, durée considérée comme un temps long. Si la question de l'impact de la durée de vie sur le développement durable est clairement posée, les travaux ayant cherché à quantifier son impact sont rares. Les travaux les plus aboutis semblent être ceux de Appu Haapio et Pertti Viitaniemi ${ }^{3}$ qui comparent différentes solutions de construction sur des échelles de temps allant de soixante à cent soixante ans. Mats Öberg ${ }^{4}$ interroge le temps pour trouver l'équilibre entre l'investissement et le coût de fonctionnement. Catarina Thormark ${ }^{5}$. a étudié comment le choix des matériaux et les possibilités de recyclage, évalués sur cinquante ans, permettent de réduire l'énergie consommée. Tarja Häkkinen ${ }^{6}$ a calculé les impacts environnementaux des enduits extérieurs et revêtements en bois pour une durée de vie de 100 ans... En dehors des travaux d'Appu Haapio, il s'agit toujours de présentation de résultats d'expériences pour lesquels jamais l'impact de la durée de vie ne semble réellement analysé. Les recherches ne paraissent pas avoir permis la production d'une modélisation articulée sur le critère de la durée et permettant l'élaboration d'outils de simulation. La durée de vie ne semble pas analysée comme une variable pouvant modifier les choix. C'est la difficulté d'évaluation et de comparaison des durées du service des différentes solutions qui explique la rareté des travaux. II y a donc un intérêt à mieux cerner ce qu'est la durée de vie d'un bâtiment et à améliorer les connaissances concernant son impact sur les composantes du développement durable.

L'objectif de cet article est de présenter synthétiquement les difficultés de prise en compte de la durée de vie des solutions et de faire émerger les outils nécessaires aux concepteurs et en particulier à l'architecte pour une prise de décision responsable. Une première partie vise à présenter les mécanismes qui régissent le vieillissement et la complexité de la durée de vie du bâtiment. Une deuxième partie présente synthétiquement les données disponibles permettant l'évaluation des impacts environnementaux et sanitaires. Enfin la dernière partie analyse les données et les normes, pose des questions et propose des pistes de recherche dont les objectifs permettraient des décisions plus performantes.

\section{Durée de vie du bâtiment Le vieillissement}

Le vieillissement associe les processus de vieillissement thermique et la réaction du produit à l'usage. Concernant le vieillissement des immeubles et des quartiers, les mécanismes de dégradations sociales et physiques ${ }^{7}$ liés au comportement des propriétaires ont été très précisément décrits. La description du phénomène " mécanique " de vieillissement des bâtiments et des villes peut aussi s'appuyer sur l'analyse démographique ${ }^{8}$. Dans le secteur du bâtiment, au travers d'un savoir-faire s'affinant et se précisant, les bâtiments ont souvent traversé des siècles. Ils ont acquis un statut particulier qui les différencie des produits courants. En France, 5,33 millions de logements avaient plus d'un siècle en 2006 (source INSEE). Les caractéristiques d'un grand nombre de bâtiments, associées à la volonté de leurs propriétaires successifs, ont permis
3. Appu Haapio, Pertti Viitaniemi, «Environmental effect of structural solutions and building materials to a building »,Environmental Impact Assessment Review, Elsevier, 2008.

4. Mats Öberg, Integrated Life Cycle Design : applied to Swedish concrete multi-dwelling buildings, thèse, Lund Institute of Technology, Lund, Suède, 2005, 307 p.
5. Catarina Thormark, «The effect of material choice on the total energy need and recycling potential of a building ", Build and Environment, 2006, vol. $41 \mathrm{n}^{\circ} 8$, p. 1019-1026.

6. Tarja Häkkinen et al, Environmental Impact of Coated Exterior Wooden Cladding, vTr, Building Technology, Finlande,1999.
7. Jean-Paul Lévy, Odile Saint Raymond, Profession propriétaire : logiques patrimoniales et logement locatif en France, Toulouse, Presses universitaires du Mirail, coll. «État des lieux », 1992.

8. Hervé Le Bras Jean-Claude Chesnais, "Cycle de l'habitat et âge des habitants", Population $n^{\circ} 2$, Paris, Presses universitaires de France / INED, 1976. 
leurs adaptations continues ou saccadées repoussant dans le temps les conséquences d'une éventuelle obsolescence. Parallèlement, de nombreux bâtiments ont volontairement ou involontairement été l'objet d'une vie plus éphémère.

\section{Durée de vie du bâtiment et facteurs d'influence}

Pour la durée, nous distinguons la vie technique du bâti et la vie fonctionnelle. Ces deux vies peuvent indépendamment entrainer la démolition

\section{Durée de vie fonctionnelle et obsolescence}

La durée de vie du bâtiment peut être affectée par les modifications fonctionnelles, c'est-à-dire des changements importants des besoins auxquels il répond. C'est le phénomène d'obsolescence. Le graphique ci-contre schématise la performance dans le temps.

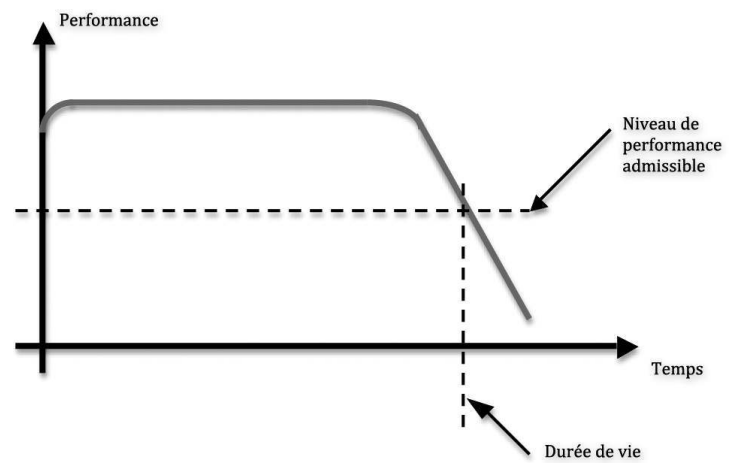

Une analyse fonctionnelle systémique permet de lister exhaustivement les fonctions du bâtiment et de présenter celles susceptibles d'en modifier la durée de vie. En résumé, les causes pouvant modifier la durée de vie et entraîner la démolition sont nombreuses. II s'agit par exemple d'un principe de précaution attaché aux catastrophes naturelles, de la disparition de toutes les activités locales ou encore de la perte de la valeur patrimoniale. Cela peut être encore un rejet total des occupants de l'image reflétée par le bâtiment et dont l'origine se trouve dans la médiocrité architecturale ou l'absence d'entretien. II peut s'agir d'un bâtiment se présentant en rupture avec la cohérence et le fonctionnement d'un îlot de la ville. Le phénomène d'obsolescence a été analysé depuis très longtemps, en particulier dans le domaine économique. Cependant, les travaux de recherche évaluant l'impact de celle-ci sur le développement durable sont rares.

\section{Durée de vie "technique" des produits et des bâtiments}

La durée de vie technique est celle résultant de l'usure liée à l'usage et à l'effet du temps. La question de l'évaluation de la durée de vie des bâtiments courants, mais surtout des produits qui les composent, est abordée depuis les années 1990. Sur la base d'une analyse structurelle et fonctionnelle des produits composant le bâtiment, les causes de défaillance sont établies et les durées de vie évaluées ${ }^{9}$. Concernant les outils d'évaluation de durée de vie, de grand progrès ont été réalisés. Les approximations statistiques des durées de vie, les méthodes et outils développés
Graphique 1.

Évolution de la

performance

de la fonction

de service.
9. Jérôme Lair, Évaluation de la durabilité des systèmes constructifs du bâtiment, thèse Génie civil, Clermont-Ferrand, Centre scientifique et technique du bâtiment, service Matériaux et laboratoire d'études et de recherches en mécaniques des structures, 2000, 213 p. Aurélie Talon, Évaluation des scénarii de dégradation des produits de construction, thèse de doctorat Génie civil, université Blaise Pascal Clermont II, 2006 
sont précis. La durée de vie technique des produits composant le bâtiment peut être évaluée avec une probabilité définie.

Une analyse systémique du bâtiment permet de produire une liste exhaustive des éléments dont la défaillance peut entrainer la démolition du bâtiment. En résumé, il semble que seuls les fondations, les murs porteurs et, dans une moindre mesure, les planchers, dont la fiabilité serait douteuse, sont susceptibles de provoquer la destruction du bâtiment. L'origine de la cause peut être interne par le biais du vieillissement ou externe par un fait accidentel. La probabilité de démolition ne se réalise que lors d'un constat de dommage généralisé. Les autres composants, dont la durée de vie est parfois très courte n'ont aucun impact direct sur la pérennité du bâtiment. C'est donc la durée de vie de la structure qui engendre la durée de vie technique du bâtiment. Aujourd'hui, l'Eurocode 0 définissant la méthode de calcul des structures préconise une durée de 50 ans pour les bâtiments courants. II est à noter que rarement les bâtiments s'effondrent d'eux-mêmes et les démolitions ayant pour cause ce risque sont très occasionnelles.

\section{Autres facteurs modifiant la durée de vie}

Lorsqu'une durée de vie est annoncée, celle-ci présuppose un entretien correct. De nombreux articles et ouvrages ont développé l'importance d'une gestion du patrimoine immobilier en ce qui concerne l'entretien ${ }^{10}$. La surveillance, l'entretien et les réparations engendrent l'extension, sous certaines conditions, de la durée de vie potentielle ${ }^{11}$.

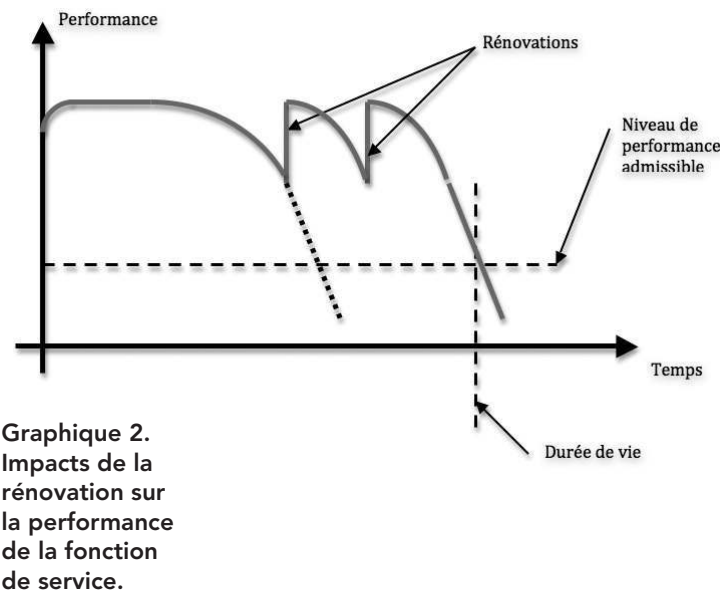

L'adaptabilité est la souplesse d'usage qui permet une adaptation du bâtiment à l'évolution des besoins. II s'agit là d'un des critères de pérennité des bâtiments. Par exemple, les locaux des berges de la Garonne construits initialement comme lieux de stockage et de bureau (XVIII siècle), devenus logements (XIX et $x x^{e}$ siècles) puis parfois bureaux et commerces puis logements ( $x x^{e}$ siècle). Enfin, le bilan effectué sur la durée de vie n'est pas identique en cas de réutilisation des matériaux lors de la démolition. La part des matériaux réutilisés ou valorisés peut venir en déduction du bilan de la construction initiale et /ou du projet pour lequel elle est réutilisée. Une observation rapide montre que les solutions techniques actuelles ne facilitent pas les possibilités de recyclage. Pour le principal, les actions se réduisent à la valorisation par intégration aux remblais autoroutiers ou à une mise en décharge.
10. Régis Bonetto, Gérard Sauce, Gestion de patrimoine immobilier. Les activités de références, СSTB, Département Technologies de l'information et Diffusion du savoir, université de Savoie, Polytech'Savoie, LOCIE, 2006.
11. Inês Flores-Colen, Jorge de Brito, "A systematic approach for maintenance budgeting of buildings façades based on predictive and preventive strategies ", Construction and Building Materials, vol. $24 n^{\circ}$ 9, Lisbonne, septembre 2010, p. $1718-1729$
12. Iso 14040. Management environnemental. Analyse du cycle de vie. Principes et cadre. 


\section{Informations disponibles pour l'évaluation des impacts}

La base de données inies contient des fiches de déclaration environnementales et sanitaires (FDES) réalisées dans le cadre de la norme NF P01-010, ellemême établie sur la base de la norme IsO série $14040^{12}$ fixant les bases de l'analyse en cycle de vie. Les valeurs établies sur l'ensemble du cycle de vie des produits (ACV) sont présentées. Ces valeurs résultent de l'addition des émissions de toutes les étapes allant de l'extraction des matières premières à la démolition du bâtiment. Seuls les impacts liés aux infrastructures de production sont négligés. L'évaluation du cycle globale est réalisée de manière précise et permet de quantifier l'impact global d'un choix. $C^{\prime}$ est cependant la comparaison entre les solutions qui intéresse le concepteur. Pour réaliser la comparaison, il est nécessaire de prendre en compte les différences de durée de vie. L'unité fonctionnelle (UF), en divisant les impacts du cycle complet par la durée de vie, doit fournir les impacts par unité de temps et permettre la comparaison. Le tableau ci-dessous présente quelques exemples des durées de vie retenues pour les calculs d'impacts.

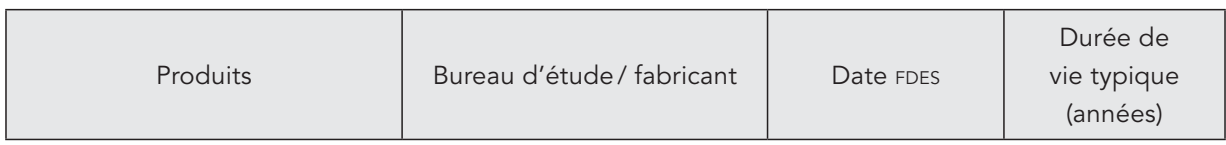

Éléments porteurs

\begin{tabular}{|l|l|l|l|}
\hline Bloc de béton creux & $\begin{array}{l}\text { CERIB / Fédération de } \\
\text { l'industrie du béton }\end{array}$ & Septembre 2006 & 100 \\
\hline $\begin{array}{l}\text { Monomur terre cuite 30 } \\
\text { rectifié }\end{array}$ & Briques de France 2006 & 150 \\
\hline Béton cellulaire $25 \mathrm{~cm}$ & $\begin{array}{l}\text { BIO IS / Syndicat national } \\
\text { des fabricants de béton } \\
\text { cellulaire }\end{array}$ & Novembre 2007 & 100 \\
\hline $\begin{array}{l}\text { Banché C25/30 CEM II avec } \\
\text { complexe de doublage } \\
\text { thermo-acoustique Ultra ThA }\end{array}$ & $\begin{array}{l}\text { ECoBILAN / Syndicat national } \\
\text { des bétons prêts à l'emploi }\end{array}$ & Septembre 2007 & 100 \\
\hline
\end{tabular}

\section{Éléments bois}

\begin{tabular}{|l|l|l|l|}
\hline $\begin{array}{l}\text { Charpente bois traditionnelle } \\
(05-027: 2009)\end{array}$ & FCBA & Juin 2009 & 100 \\
\hline $\begin{array}{l}\text { Panneau de lamelles de bois } \\
\text { OBS (Oriented Strand Board) } \\
N^{\circ} 05-020: 2009\end{array}$ & FCBA & Mars 2009 & 100 \\
\hline
\end{tabular}

\footnotetext{
Tableau. Fiche

de déclaration

environnementale

et sanitaire, FDES.

Exemples de durées

de vie typique.
} 
Bien que les matériaux soient très différents, les durées de vie typiques sont identiques. Pourtant, si I'on prend l'exemple des charpentes, il en existe qui ont rempli leurs fonctions plusieurs siècles. Pourquoi prend-on une durée de 100 ans ?

\section{Analyse et synthèse}

La première partie montre que la durée de vie d'un bâtiment est variable. II est donc nécessaire de mieux connaître l'impact de cette durée sur les composantes du développement durable. Pour cette raison, analysons les points soulevant des difficultés.

\section{Conception et obsolescence}

Concernant les bâtiments courants, la durée de vie d'un bâtiment est davantage liée à la fonction du bâtiment qu'à ses performances techniques dans le temps. Plus un bâtiment est souple dans son fonctionnement, plus il est apte à s'adapter à l'évolution. Ici, c'est la qualité de travail du concepteur qui permettra l'adaptation à l'évolution des besoins dans le temps et réduira le risque de démolition. Il s'agit d'un " recyclage » fonctionnel du bâtiment. Quelle est la performance de cette souplesse fonctionnelle en termes de développement durable? Quels coûts économiques, environnementaux et sociaux, leur mise aux standards de confort, de sécurité et leur entretien permettent de leur prolonger la vie?

II semble que peu d'études aient été réalisées sur les fondements de ces caractéristiques. Peu d'outils méthodologiques de conception, de simulation ou d'évaluation ont été développés pour ce critère de durée de vie.

\section{Durée de vie des matériaux et des bâtiments}

L'analyse en cycle de vie (ACV) permet une grande avancée dans la prise en compte des impacts des choix du concepteur. Les normes NF 01-01013 et IsO $15686^{14}$ sont précieuses car elles fixent un cadre précis permettant les évaluations. Les valeurs des impacts sur le cycle de vie complet sont précises. Néanmoins, les Uf nécessaires à la comparaison des solutions entre-elles ne peuvent permettre de conclure. La durée de vie typique représente la durée de vie du produit dans l'ouvrage étudié et n'a pas de lien direct avec la durée de vie réelle ou même théorique du produit. Prendre des durées quasi forfaitaires, identiques d'une solution à l'autre, ne permet ni d'aider au choix en fonction de performances réelles ni d'évaluer si le choix doit être réalisé en fonction de la durée attendue du projet. Le projet actuel de décret-arrêté de "Déclaration environnementale produits " ne semble pas attendre pour ce qu'il en est connu, une plus grande précision dans l'établissement des durées de vie. Les évaluations de durées "techniques " sont encore trop peu nombreuses. C'est dans la catégorie des composants de la structure des bâtiments que l'information manque particulièrement. Les recherches et les évaluations sont davantage réalisées sur les équipements au cycle de vie court. Le programme PREBAT-EVA, ambitieux et qui aurait pu fournir de précieuses informations, ne paraît pas devoir être finalisé.

En 2006, I'INSEE comptabilisait 12 millions de logements ayant plus de cinquante ans et 5,33 millions de logements ayant plus d'un siècle. Ces chiffres importants ne peuvent

13. NF 01-010: norme française définissant le contenu et le mode de réalisation de la fiche de déclaration environnementale et sanitaire dans le cadre des produits de construction.

14. Iso 15686-1: 2000. Buildings and constructed assets. Service life planning. Part 1: General principles. 
pas être le résultat d'un hasard. Comment l'Eurocode 0 peut-il avoir déterminé une valeur de cinquante ans pour le calcul des structures ? Cette valeur est le résultat de calculs probabilistes de fiabilité. Résulte-t-elle d'une optimisation des coûts économiques, environnementaux et sociaux ? Ne devrait-elle pas être le résultat d'une volonté collective de la société civile ? Il est probable que cette durée "normalisée » devienne un objectif pour les professionnels. Dans ce cas, satisfera-t-elle les maîtres d'ouvrage?

Enfin, dans le cadre d'une comparaison de solutions techniques, l'évaluation de l'impact de la durée de vie sur le développement doit nécessairement intégrer les impacts des actions d'entretien ayant permis la performance de longévité.

\section{L'architecte dans son rôle}

Compte tenu de sa place centrale dans le projet, de sa culture globale et transversale et de sa capacité à prévoir l'évolution du bâti dans sa réponse au besoin, l'architecte est le premier acteur susceptible de prendre en compte le paramètre du temps. Lorsqu'il répond à la demande du maître d'ouvrage, il doit donc s'interroger. S'agit-il d'un projet ayant le caractère d'un bien de consommation courant ? Participera t-il au patrimoine personnel du maître d'ouvrage ? Atteindra-t-il le statut d'élément de patrimoine culturel pour la société ? Doit-il répondre strictement au besoin exprimé ou en envisager l'évolution ? Quels choix techniques permettront de fournir le service attendu sur une durée désirée au moindre coût global " étendu » ? En vue de l'optimisation et de l'amélioration de la performance en termes de développement durable, l'architecte doit pouvoir répondre à ces questions. Pour enrichir sa réponse architecturale, ce professionnel a besoin d'informations et d'outils d'aide à la décision. Ces questions ne sont pas un frein à la création, mais peuvent au contraire enrichir la réponse architecturale considérant le critère temporel.

\section{Conclusion}

Les questions mises en évidence dans cet article sont les suivantes :

- quel est l'impact de la durée de vie du bâtiment et des solutions techniques sur le développement durable?

- quelles solutions techniques doit-on choisir pour une durée envisagée en vue de l'optimisation d'une approche en termes de développement durable?

Afin de pouvoir répondre à ces questions et d'aider la production d'outils, de méthodes d'évaluation ou de grilles d'analyse, il paraît important d'analyser auparavant en quoi les bâtiments se distinguent des biens de consommation courante. Avec cet objectif, les points suivants ne peuvent échapper à un travail d'analyse. Les durées de vie mentionnées dans l'Eurocode sont un sujet de questionnement quant aux raisons qui justifient les valeurs retenues. Ces valeurs sont d'autant plus importantes que présentées comme des durées " normalisées ", elles deviendront rapidement des objectifs pour les professionnels. Dans les bases de données à vocation environnementale et sanitaire, les durées de vie paraissent établies de manière forfaitaire et souvent sans fondement. Différentes techniques permettent aujourd'hui d'évaluer la durée de vie des 
composants du bâtiment. II est nécessaire de mieux connaître la durée de vie technique des produits composant la structure des bâtiments afin de pouvoir comparer les choix. La production d'outils modélisant les impacts de la durée de vie des bâtiments sur le développement durable faciliterait la prise de décision. Pour réaliser de justes comparaisons de solutions techniques, ces outils doivent intégrer les impacts des interventions d'entretien qui prolongent la durée de vie. En leur absence, il ne peut y avoir une réelle optimisation de développement durable. De tels outils permettraient aussi d'optimiser la performance d'un bâtiment confronté à une éventuelle rénovation ou démolition en fonction des solutions originelles retenues.

Les facteurs favorisant l'obsolescence, I'accroissement de la valeur patrimoniale et les causes sociétales de fin de vie, ne semblent pas avoir fait l'objet de véritables études. L'architecte responsable de l'adaptabilité des bâtiments à l'évolution des besoins ne peut ignorer ces facteurs. L'analyse approfondie des caractéristiques d'adaptabilité à l'évolution des besoins paraît une des conditions à la pérennité. L'analyse des causes de vieillissement fonctionnel, associé au comportement humain, serait sans doute pleine d'enseignement. Les concepteurs ont donc besoin d'outils d'aide à la décision intégrant le paramètre de la durée de vie dans les évaluations d'impacts. 